\title{
Teste das microbolhas estáveis no líquido amniótico de recém-nascidos prematuros para predição da maturidade pulmonar
}

\author{
Stable microbubble test on amniotic fluid of premature newborns \\ for lung maturity prediction \\ Prueba de las microbolhas estables en el líquido amniótico de recién \\ nacidos prematuros para predicción de la madurez pulmonar \\ Márcio da Silva PEREIRA ${ }^{1}$ \\ Dayse Caroline Martírios LUZ ${ }^{2}$ \\ Roberto Eduardo Donoso RIVERO ${ }^{3}$ \\ Michaella Fernandes ALENCAR \\ Silvana Céspedes GÓMEZ ${ }^{5}$ \\ Gloria Liz Becerra NACCHA \\ Ana Maria Viola RENSI ${ }^{6}$ \\ Ana Flávia Moura MENDES ${ }^{7}$ \\ Cláudio Marcio Fiorenza de SOUZA ${ }^{8}$ \\ Maria Rita Carvalho de Freitas AMORIM ${ }^{9}$ \\ Arthur Luiz Carvalho de AMORIM ${ }^{9}$ \\ Paulo Brandão Sakr KHOURI 10 \\ Lucas Amaral DRUZIAN ${ }^{\mathbf{1 1}}$ \\ ${ }^{1}$ Médico graduado pelo Centro Universitário Christus (UNICHRISTUS), 60190-060, Fortaleza - Ceará, Brasil. \\ ${ }^{2}$ Médica graduada pela Faculdade Integral Diferencial (FACID), 64052-410, Teresina PI, Brasil. \\ ${ }^{3}$ Médico graduado pela Universidad Autónoma Gabriel René Moreno (UAGRM), Santa Cruz de la Sierra, Bolívia. \\ ${ }^{4}$ Médica graduada pela Universidade Federal do Pernambuco (UFPE), 50670-901, Recife-Pernambuco, Brasil. \\ ${ }^{5}$ Médica graduada pela Universidad de San Martin de Porres, 15009, Lima Peru. \\ ${ }^{6}$ Médica graduada pela Universidade Brasil (UB), 15600-000, Fernandópolis - São Paulo, Brasil. \\ ${ }^{7}$ Médica graduada pela Universidade José do Rosário Vellano (UNIFENAS), 31710-030, Belo Horizonte Minas Gerais, Brasil. \\ ${ }^{8}$ Médico graduado pela Faculdades Integradas Aparício Carvalho (FIMCA), 76980-000, Porto Velho RO, Brasil. \\ ${ }^{9}$ Médica graduada pela Universidade Potiguar (UNP), 59020-010, Natal - Rio Grande do Norte, Brasil. \\ ${ }^{10}$ Acadêmico de Medicina da Universidade Católica de Brasília (UCB), 71966-700, Brasília - DF, Brasil \\ ${ }^{11}$ Médico graduado pela Pontifícia Universidade Católica de Goiás (PUC-GO), 74175-120, Goiânia - GO, Brasil
}

\section{Resumo}

Introdução À medida que o tratamento profilático ou precoce com surfactante mostrou-se vantajoso nos recém-nascidos prematuros com imaturidade pulmonar renovou-se o interesse na busca de testes diagnósticos nesta área. Objetivo Avaliar o desempenho do TME no aspirado gástrico, na predição da síndrome do desconforto respiratório (SDR) em recém-nascidos prematuros. Material e Método: Foi coletado aspirado gástrico em partos prematuros ocorridos no Hospital Geral Dr. César Cals (HGCC), no período compreendido entre julho de 2015 a julho de 2017 e realizado o TME nesse material. Resultados A idade média dos recém-nascidos foi de foi de 30,02 $\pm 1,98$ semanas e a média do peso e o desvio padrão foi 2019,50 $\pm 573,2$ gramas. Considerando-se importâncias iguais para sensibilidade e especificidade, o corte de melhor desempenho para o TME foi de $9 \mu \mathrm{b} / \mathrm{mm} 2$. Levando em consideração esse ponto de corte, a sensibilidade encontrada foi de 100\% (IC 95\%: 96 -100) e a especificidade de 90\% (IC 95\%: 68-99). Conclusão: O TME no aspirado gástrico de prematuros tem alta sensibilidade e especificidade para predizer a SDR. O valor da sensibilidade encontrado neste estudo (100\%) foi o maior já relatado na literatura mundial, levando-se em consideração a utilização de apenas um único teste (TME) em diferentes amostras. O TME no aspirado gástrico apresenta um excelente desempenho para predizer a SDR, similar aos demais testes mais sofisticados descritos na literatura, como por exemplo, a contagem de corpos lamelares.

Descritores: Técnicas e Procedimentos Diagnósticos; Proteínas Associadas a Surfactantes Pulmonares; Líquido Amniótico; RecémNascido Prematuro.

\section{Abstract}

Introduction As prophylactic or early treatment with surfactant was shown to be advantageous in preterm infants with pulmonary immaturity, there was renewed interest in the search for diagnostic tests in this area. Objective To evaluate the performance of TME in gastric aspirate in the prediction of respiratory distress syndrome (RDS) in preterm infants. Methods Gastric aspirate was collected in preterm births at the General Hospital Dr. César Cals (HGCC) in the period from July 2015 to July 2017 and the TME was performed in this material. Results The mean age of the newborns was of $30.02 \pm 1.98$ weeks and the mean weight and standard deviation was $2019.50 \pm$ 573.2 grams. Considering equal amounts for sensitivity and specificity, the best cutoff for TME was $9 \mu \mathrm{b} / \mathrm{mm} 2$. Taking this cut-off point into account, the sensitivity was 100\% (95\% CI: 96 -100) and the specificity was 90\% (95\% CI: 68-99). Discussion The present study showed that the SMT in gastric aspirate of preterm infants has high sensitivity and specificity to predict RDS. In the authors' knowledge, the value of the sensitivity found in this study $(100 \%)$ was the largest ever reported in the world literature, taking into account the use of only one test (TME) in different samples. Conclusion We concluded that TME in the gastric aspirate presents an excellent performance to predict the RDS, similar to the other more sophisticated tests described in the literature, such as, for example, the count of lamellar bodies. Descriptors: Diagnostic Techniques and Procedures; Pulmonary Surfactant-Associated Proteins; Amniotic Fluid; Infant, Premature.

\section{Resumen}

Introducción A medida que el tratamiento profiláctico o precoz con surfactante se mostró ventajoso en los recién nacidos prematuros con inmadurez pulmonar, se renovó el interés en la búsqueda de pruebas diagnósticas en esta área. Objetivo Evaluar el desempeño del TME en el aspirado gástrico, en la predicción del síndrome de la incomodidad respiratoria (SDR) en recién nacidos prematuros. Métodos Se recogió aspirado gástrico en partos prematuros ocurridos en el Hospital General Dr. César Cals (HGCC), en el período comprendido entre julio de 2015 a julio de 2017 y realizado el TME en ese material. Resultados La edad media de los recién nacidos fue de 30,02 $\pm 1,98$ semanas y la media del peso y la desviación estándar fue de 2019,50 $\pm 573,2$ gramos. Se consideró que las cantidades iguales para sensibilidad y especificidad, el corte de mejor desempeño para el TME fue de $9 \mu \mathrm{b} / \mathrm{mm} 2$. La sensibilidad encontrada fue del 100\% (IC 95\%: 96 -100) y la especificidad del 90\% (IC 95\%: 68-99). Discusión El presente estudio mostró que el TME en el aspirado gástrico de prematuros tiene alta sensibilidad y especificidad para predecir la SDR. En el conocimiento de los autores, el valor de la sensibilidad encontrado en este estudio (100\%) fue el mayor ya relatado en la literatura mundial, teniendo en cuenta la utilización de sólo una sola prueba (TME) en diferentes muestras. Conclusión Concluimos que el TME en el aspirado gástrico presenta un excelente desempeño para predecir la SDR, similar a las demás pruebas más sofisticadas descritas en la literatura, como por ejemplo, el conteo de cuerpos lamelares.

Descriptores: Técnicas y Procedimientos Diagnósticos; Proteínas Asociadas a Surfactante Pulmonar; Líquido Amniótico; Recien Nacido Prematuro.

\section{INTRODUÇÃO}

O surfactante pulmonar é uma substância fundamental na mecânica pulmonar. É composto por fosfolípides e proteínas que reduzem a tensão superficial na interface ar /líquido do alvéolo, evitando assim o colabamento durante a expiração final.
Também participa na defesa inata do hospedeiro contra patógenos inalatórios ${ }^{1}$.

Nos últimos anos, testes de avaliação da maturidade pulmonar vêm sendo estudados para que não seja administrado surfactante exógeno a todos os prematuros com 
menos de 32 semanas de gestação, mas somente àqueles que apresentem testes indicando imaturidade pulmonar. A esses pacientes seria, então, administrado surfactante precocemente, assim que os testes demonstrassem imaturidade e antes que o paciente apresentasse sinais de sofrimento respiratório ${ }^{2}$.

Os testes usados para avaliação da maturidade pulmonar quantificam a presença do surfactante ou analisam qualitativamente a sua função em 3 principais meios: líquido amniótico, no aspirado gástrico e no aspirado traqueal. Dentre os testes existentes destaca-se o Teste das Microbolhas Estáveis (TME), criado por Pattle, et al. ${ }^{3}$ Trata-se de um teste rápido (5 a 10 minutos), com baixos custos e de fácil operacionalização, que verifica a capacidade das secreções que contêm surfactante em formar bolhas estáveis com menos de 15 micrômetros ${ }^{3}$.

O presente estudo tem por objetivo avaliar se o TME no líquido amniótico de recém-nascidos (RN) com e sem SDR apresenta suficiente acurácia para predizer a síndrome do desconforto respiratório em $\mathrm{RN}$ prematuros. Secundariamente pretende-se estabelecer o melhor ponto de corte no TME, ou valor de referência, para predizer necessidade de surfactante e comparar a acurácia do TME entre os grupos de prematuros para predizer a grau de maturidade pulmonar.

\section{MATERIAL E MÉTODO}

Trata-se de um estudo observacional de coorte. A pesquisa foi realizada na Unidade de Tratamento Intensiva Neonatal do Hospital Geral Dr. César Cals (HGCC), no período compreendido entre julho de 2015 a julho de 2017.

Mediante amostra de conveniência, foram incluídos pacientes com idade gestacional inferior a 37 semanas de evolução, dos quais foi possível coletar líquido amniótico no máximo 48 horas antes do nascimento.

Foram excluídos os recém-nascidos prematuros com pneumonia, cardiopatias congênitas, pneumotórax, hérnia diafragmática e/ou síndromes genéticas que levassem a algum outro tipo de comprometimento respiratório, casos em que não foi possível definir o diagnóstico de SDR de acordo com os critérios diagnósticos utilizados no estudo; e líquido amniótico com mecônio espesso ${ }^{3}$.

O diagnóstico de SDR foi feito de acordo com critérios clínicos (taquipneia, retrações costais e esternal, gemência, cianose em ar ambiente, necessidade de uma $\mathrm{FiO} 2$ maior que 0,4 ) associados a um padrão radiológico compatível (aspecto retículo-granulado difuso e presença de broncogramas aéreos) ${ }^{4}$.

Determinou-se a IG ao nascimento de acordo com a data da última menstruação e confirmada por ultrassom fetal precoce (antes de 20 semanas). Nos casos em que nenhum desses dados estivessem disponíveis ou que fossem confiáveis, considerou-se, então, o exame do RNPT logo após o nascimento com a escala de New Ballar ${ }^{1}$.

Os membros da equipe de obstetrícia do HGCC foram orientados a coletar líquido amniótico das pacientes em trabalho de parto prematuro, seja por via vaginal ou após a histerotomia nos partos cesáreos ${ }^{4}$.

O líquido amniótico foi armazenado em refrigerador por período de até 24 horas e transferido para congelador a $20^{\circ} \mathrm{C}$ até a realização do TME. O processo de congelamento foi validado em estudo anterior. A presença ou ausência de SDR e os demais dados foram coletados pelo autor através de protocolo desenvolvido para este estudo após 72 horas do nascimento ${ }^{5}$. Foram registrados de cada recém-nascido participante da amostra:

- Identificação do paciente (nome, gênero, número do prontuário);

- Dados do nascimento (data e hora, índice de Apgar no $1^{\circ}$ e $5^{\circ}$ minutos, peso);

- Idade gestacional (baseada em ecografia obstétrica se realizada antes de 20 semanas, DUM ou New Ballard);

- História materna (idade, antecedentes obstétricos, tempo da administração de corticoide pré-natal, uso de medicações, doenças prévias);

- Tipo de parto (normal ou cesariana);

- Valor do Teste de Microbolhas Estáveis;

- Desenvolvimento ou não de SDR;

- Uso de surfactante exógeno;

- Uso de CPAP ou ventilação mecânica (e tempo de permanência, caso houvesse resposta afirmativa para qualquer uma das alternativas) ${ }^{4}$.

$\mathrm{Na}$ análise do líquido amniótico, uma quantidade mínima de $40 \mu \mathrm{l}$ foi aspirada e expelida rapidamente, cerca de 20 vezes (duração aproximada de 6 segundos) sobre uma câmara de contagem (Neubauer Improved Bright-Line), por meio de uma pipeta de Pasteur, adaptada a uma pera de borracha de $2 \mathrm{ml}$. A câmara foi rapidamente invertida, formando uma gota e, após quatro minutos, o número de microbolhas foi contado em um microscópio de luz, utilizando uma objetiva de 10x. Foram contadas, em $1 \mathrm{~mm}^{2}$, somente as microbolhas com diâmetro menor que $15 \mu \mathrm{m}$ (Figura 1) 3 .5

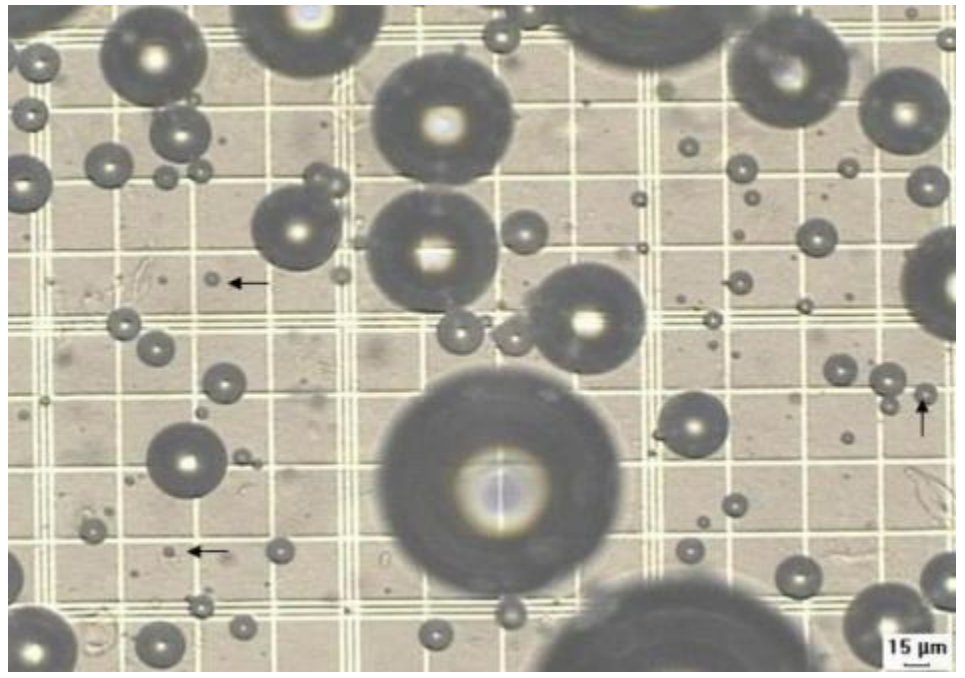

Figura 1: Contagem microscópica das microbolhas. As setas indicam microbolhas com menos de $15 \mu \mathrm{m}^{3,5}$

Para a descrição das variáveis quantitativas e qualitativas, utilizou-se estatística descritiva, como a média, o desvio padrão, a mediana, o mínimo e o máximo.

Para se verificar se existem diferenças entre os grupos com e sem SDR, aplicou-se o teste $t$ para variáveis quantitativas e o teste da mediana para variáveis qualitativas.

Para a predição de SDR se utilizou o modelo de regressão logística com o TME como variável preditora. Para verificar a acurácia dos modelos ajustados, estimou-se, por ponto e intervalo, as áreas sob a curva ROC. Com base nesta curva, definiu-se o melhor ponto de corte para o teste ${ }^{2}$.

O nível de significância adotado foi de $5 \%$ e os cálculos foram realizados com auxílio dos programas SPSS 17.0, PEPI 4.0 e o Epi Info 6.0.

O projeto do estudo em questão foi submetido ao Comitê de Ética em Pesquisa do Centro Universitário Christus. O Centro de Estudos e Pesquisa do Hospital Geral Dr. César Cals (HGCC) foi informado sobre o estudo, e recebeu cópia do projeto de pesquisa e um termo solicitando a autorização da pesquisa. A concordância aos termos expostos no projeto e a aprovação pelo Centro de Estudos foi efetivada mediante a assinatura do Superintendente Técnico da Instituição no parecer consubstanciado fornecido pelo setor responsável do hospital.

\section{RESULTADOS}

No total, 72 pacientes tiveram líquido amniótico coletado. Foram excluídos da amostra: 17 recém-nascidos devido à falta de critérios diagnósticos para SDR e 15 por apresentarem mecônio espesso. Restaram 40 pacientes para análise, sendo que 10 (25\%) apresentaram critério diagnóstico para SDR. A idade média dos recém-nascidos foi de 30,02 \pm 
1,98 semanas e a média do peso e o desvio padrão foi 2019,50 $\pm 573,2$ gramas.

Os escores de Apgar foram significativamente menores no grupo com desconforto respiratório: RNPT com SDR (Média: 7/8) e RNPT sem SDR (Média: 9/9). Dos 20 pacientes com SDR, 17 foram tratadas com Pressão Positiva Contínua de Vias Aéreas, 15 necessitaram de ventilação mecânica e 19 receberam surfactante.

Quanto a presença de doenças maternas prévias, 15 $(37,5 \%)$ possuíam Infecção do Trato Urinário, 10 (25\%) Diabetes Mellitus, 7 (17,5\%) Hipertensão Arterial Sistêmica e $25 \%$ Descolamento Prematuro da Placenta, totalizando $85 \%$ da amostra estudada. O resumo dos dados de caracterização da amostra é descrito na Tabela 1.

A curva ROC foi feita para auxiliar na avaliação do melhor ponto de corte do TME para predição da maturidade pulmonar, e, por conseguinte, prevê a necessidade da terapia com surfactante exógeno ${ }^{6}$.

Como pode ser observado na Figura 2 há uma grande área sob a curva, indicando uma boa acurácia diagnóstica $(0,987$ - IC95\%: 0,964 - 1,000). Utilizando-se um ponto de corte de 9 corpos lamelares/ $\mu$ l obteve-se sensibilidade de 100\% (IC 95\%:96 -100) e especificidade de 90\% (IC 95\%: 68-99).

Tabela 1. Características dos pacientes ao nascimento. Resultados expressos em média (desvio padrão), nas variáveis contínuas, ou em número de pacientes (percentual)

\begin{tabular}{lcc}
\hline & $\begin{array}{l}\text { SDR } \\
(\mathbf{N = 2 0 )}\end{array}$ & $\begin{array}{l}\text { Sem SDR } \\
(\mathbf{N = 2 0 )}\end{array}$ \\
\hline Idade Gestacional & $27,35 \pm 2,123$ & $32,70 \pm$ \\
& & 1,849 \\
Peso & $1765,50 \pm 518,6$ & $2273,50 \pm$ \\
& & 627,8 \\
Sexo Masculino & $12(60 \%)$ & $10(50 \%)$ \\
Corticóide & $7(35 \%)$ & $0(0 \%)$ \\
Cesariana & $18(90 \%)$ & $12(60 \%)$ \\
& Doenças Prévias & \\
ITU & $8(40 \%)$ & $6(30 \%)$ \\
DM & $6(30 \%)$ & $4(20 \%)$ \\
HAS & $4(20 \%)$ & $3(15 \%)$ \\
\hline DPP & $\mathbf{o ( o \% )}$ & $\mathbf{2 ( 1 0 \% )}$ \\
\hline
\end{tabular}

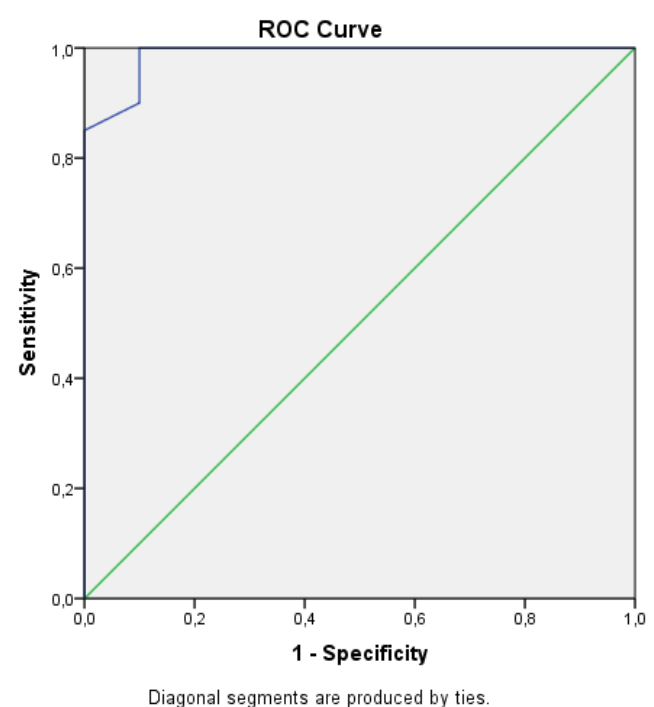

Figura 2: Curva ROC com os respectivos valores de sensibilidade e especificidade do TME.

Um paciente com SDR apresentou resultados erroneamente positivos para maturidade pulmonar (falso negativo) e, no grupo que não desenvolveu SDR, dois pacientes apresentaram teste erroneamente negativo para maturidade pulmonar (falso positivo).

Para a análise dos valores de predição desse método diagnóstico, foram testados, além dos parâmetros de sensibilidade (S) e especificidade (E), o valor preditivo positivo (VPP) e o valor preditivo negativo (VPN).

O Valor Preditivo Positivo encontrado foi de $90 \%$ e Valor Preditivo Negativo de $100 \%$. Ambos os valores prestaram-se para a avaliação da confiabilidade dos resultados das provas e foram calculados mediante as seguintes equações: VPP $=$ [verdadeiro-positivos/ (verdadeiro-positivos + falso-positivos)] $\mathrm{x}$ 100\%; VPN $=$ [verdadeironegativos/(verdadeiro-negativos + falso-negativos) $] \times 100 \%{ }^{6}$.

\section{DISCUSSÃO}

O presente estudo mostrou que o TME no líquido amniótico de prematuros tem alta sensibilidade e especificidade para predizer a SDR. No conhecimento dos autores, o valor da sensibilidade encontrado neste estudo (100\%) foi o maior já relatado na literatura mundial, levandose em consideração a utilização de apenas um único teste (TME).

A contagem de corpos lamelares no líquido amniótico, descrita por Dubin para avaliação de maturidade pulmonar fetal, é um teste rápido, de baixo custo e possível de ser realizado com pequeno volume de amostra. Sua utilidade para determinar a maturidade pulmonar tem sido descrita em vários estudos realizados com líquido amniótico ${ }^{7}$.

O primeiro estudo que analisava os resultados do TME e sua relação com a SDR, além de compará-lo com a Relação L/E e com o Teste de Clements, foi publicado por Pattle et $\mathrm{al}^{3}{ }^{3}$ em 1979. Esse estudo mostrou que contagem de mais de 20 microbolhas estáveis por milímetro quadrado $\left(\mathrm{mbe} / \mathrm{mm}^{2}\right)$ no TMBE apontava maturidade pulmonar. Pelo fato de o estudo ter comparado o TME com a Relação L/E, Pattle et al. ${ }^{3}$ sugeriram que fosse dispensada a realização do teste $\mathrm{L} / \mathrm{E}$, quando contagens elevadas de microbolhas estáveis fossem encontradas.

Teeratakulpisarn et al. $^{8}$ realizaram o teste com aspirado gástrico de recém-nascidos, utilizando um ponto de corte de 20 microbolhas estáveis por milímetro quadrado $\left(\mathrm{mbe} / \mathrm{mm}^{2}\right)$, e encontraram sensibilidade e especificidade em torno de $95 \%$ e valor preditivo negativo acima de $99 \%$ para identificar recém-nascidos com deficiência de surfactante.

No estudo de Boo et al. ${ }^{9}$ foram analisadas 104 amostras de aspirado traqueal, sendo que o TME igual ou menor a $70 \mathrm{mbe} / \mathrm{mm}^{2}$ teve a maior acurácia para $\mathrm{o}$ diagnóstico de SDR, com uma sensibilidade de 97,7\% e especificidade de 90,3\%. A grande limitação para uso de fluido traqueal é a necessidade de entubação e a complexidade para obter a amostra.

Mehrpisheh et al. ${ }^{10}$ verificaram correlação significativa entre a atividade surfactante, evidenciada pela porcentagem de microbolhas na amostra e a severidade da SDR. Quando foi utilizado um ponto de corte de $10 \mathrm{mbe} / \mathrm{mm}^{2}$ ou menos para definir o diagnóstico de SDR, a sensibilidade foi de $73,9 \%$ e a especificidade de $92 \%$.

No presente estudo, pudemos constatar que a maioria dos lactentes atermos com dificuldade respiratória tiveram uma baixa contagem de MBE enquanto que os RN sem desconforto respiratório obtiveram contagens elevadas de MBE, usando como melhor ponto de corte $9 \mu \mathrm{b} / \mathrm{mm} 2$ (Sensibilidade de $100 \%$, especificidade de $90 \%$ ).

A partir dessa constatação foi possível se prever corretamente a necessidade de surfactante em 19 dos 20 recém-nascidos pré-termos inclusos no grupo com SDR, e que, portanto, vieram a desenvolver SDR posteriormente. Já no grupo dos recém-nascidos sem SDR, apenas 2 necessitaram posteriormente de surfactante exógeno, devido a evolução desfavorável do quadro clínico.

Um fator relevante do estudo é que os 2 recém nascidos considerados como falsos positivos no TME, ou seja, que tiveram teste indicando imaturidade pulmonar, porém não desenvolveram o quadro característico da SDR, posteriormente vieram a desenvolver algum grau de disfunção respiratória necessitando de pelo menos 24 horas de CPAP.

O TME no líquido amniótico é preciso para o diagnóstico de SDR e pode ser útil para apoiar a decisão de administrar surfactante nos recém-nascidos pré-termos ${ }^{10}$.

Muitas questões sobre o possível uso clínico deste 
teste ainda precisam ser respondidas por pesquisas futuras que englobem um número maior de pacientes para que se possa determinar com maior precisão os melhores pontos de corte do teste e para que futuramente seja envidada a possibilidade de utilizar esse teste nas UTI neonatais de todo o Brasil, a exemplo do que já ocorre em maternidades do Rio Grande do $\mathrm{Sul}^{5}$.

\section{CONCLUSÃO}

O TME no aspirado gástrico mostrou alta sensibilidade e excelente desempenho para predizer a SDR, similar aos demais testes mais sofisticados descritos na literatura, como por exemplo, a contagem de corpos lamelares. $\mathrm{O}$ valor da sensibilidade encontrado neste estudo (100\%) foi o maior já relatado na literatura mundial, levando-se em consideração a utilização de apenas um único teste (TME) em diferentes amostras.

\section{REFERÊNCIAS}

1. Eckert Seitz E, Fiori HH, Luz JH, Fiori RM. Stable microbubble test on tracheal aspirate for the diagnosis of respiratory distress syndrome. Biol Neonate. 2005; 87(2):140-4.

2. Reuter S, Moser C, Baack M. Respiratory distress in the newborn. Pediatr Rev. 2014; 35(10):417-28.

3. Pattle RE, Kratzing CC, Parkinson CE, Graves L, Robertson RD, Robards GJ et al. Maturity of fetal lungs tested by production of stable microbubbles in amniotic fluid. Br J Obstet Gynaecol. 1979; 86(8):615-22.

4. Roiz-Hernandez J, Navarro-Solis E, Carreon-Valdez E. Lamellar bodies as a diagnostic test of fetal lung maturity. Int J Gynaecol Obstet. 2002;77(3):217-21.

5. Fiori HH. Profilaxia Seletiva com Surfactante em Prematuros Nascidos com $\leq 31$ Semanas de Idade Gestacional Usando o Teste de Microbolhas Estáveis no Aspirado Gástrico [tese]. Porto Alegre: Pontifícia Universidade Católica do Rio Grande do Sul, Faculdade de Medicina-PUCRS; 2005.

6. Pattle RE. Properties, function and origin of the alveolar lining layer. Nature. 1955; 175(4469):1125-6.

7. Dubin SB. Characterization of amniotic fluid lamellar bodies by resistivepulse counting: relationship to measures of fetal lung maturity. Clin Chem. 1989; 35(4):612-6.

8. Teeratakulpisarn J, Taksaphan S, Pengsaa K, Wiangnon S, Kosuwon W. Prediction of idiopathic respiratory distress syndrome by the stable microbubble test on gastric aspirate. Pediatr Pulmonol. 1998; 25(6):383-9.

9. Boo NY, Cheong KB, Cheong SK, Lye MS, Zulfiqar MA. Usefulness of stable microbubble test of tracheal aspirate for the diagnosis of neonatal respiratory distress syndrome. J Paediatr Child Health. 1997; 33(4):329-34.

10. Mehrpisheh S, Mosayebi Z, Memarian A, Kadivar M, Nariman S, Ostadrahimi P et al. Evaluation of specificity and sensitivity of gastric aspirate shake test to predict surfactant deficiency in Iranian premature infants. Pregnancy Hipertens. 2015;5(2):182-6.

\section{CONFLITO DE INTERESSES}

Os autores declaram não haver conflitos de interesse.

\section{AUTOR PARA CORRESPONDÊNCIA}

Márcio da Silva Pereira

marcio7x@hotmail.com

Submetido em 21/12/2017

Aceito em 17/01/2018 